\title{
Telemedicine information system for patients with amputated limbs
}

\author{
Monica Dascalu ${ }^{1,4}$, Mihail Stefan Teodorescu ${ }^{1}$, Adrian Barbilian ${ }^{2}$, Elteto Zoltan ${ }^{4}$, Madalina Streinu $^{2}$, \\ Mark Edward Pogarasteanu ${ }^{3}$, Lucian Milea ${ }^{1}$, Anca Plavitu ${ }^{4,6}$, Dan Coroama ${ }^{1}$, Eduard Franti ${ }^{4,5}$ \\ ${ }^{1}$ Faculty of Electronics and Telecommunications, Politehnica University of Bucharest, Bucharest, Romania \\ ${ }^{2}$ Faculty of Medicine, "Carol Davila" University of Medicine and Pharmacy, Bucharest, Romania \\ 3“"Dr. Carol Davila" Central Military University Emergency Hospital, Bucharest, Romania \\ ${ }^{4}$ Centre for New Electronic Architectures, Research Institute for Artificial Intelligence, Bucharest, Romania \\ ${ }^{5}$ Simulation, Modelling and Computer Aided Design Laboratory, IMT Bucharest, Romania \\ ${ }^{6}$ Faculty of Exact Sciences and Engineering, Hyperion University of Bucharest, Bucharest, Romania
}

\section{Email address:}

monicad@artsoc.ro (M. Dascalu), mihait@artsoc.ro (M. Teodorescu), adrian@barbilian.ro(A. Barbilian), zelteto@gmail.com (Z. Elteto), mark.pogarasteanu@gmail.com (M. Pogarasteanu), madalinas@artsoc.ro (M. Streinu), lucian@artsoc.ro (L. Milea), ancap@artsoc.ro (A. Plavitu),dcoroama@artsoc.ro (D. Coroama), edif@artsoc.ro (E. Franti)

\section{To cite this article:}

Monica Dascalu, Mihail Stefan Teodorescu, Adrian Barbilian, Elteto Zoltan, Madalina Streinu, Mark Edward Pogarasteanu, Lucian Milea, Anca Plavitu, Dan Coroama, Eduard Franti. Telemedicine Information System for Patients with Amputated Limbs. International Journal of Biomedical Science and Engineering. Vol. 2, No. 5, 2014, pp. 45-51. doi: 10.11648/j.ijbse.20140205.12

\begin{abstract}
The paper describes the implementation of a telemedicine platform that is developed in collaboration by a multidisciplinary team of researchers (engineers and doctors). The complex telemedicine information system aims to offer distance medical assistance to patients with amputated limbs mainly for those using prosthetic limbs. Although not intended to replace the classical relationship doctor-patient and the direct human interaction, the telemedicine system is an important tool in special cases when the patients live in remote areas or do not have the possibility to go to the hospital. During the training procedure often necessary for the performant use of a prosthesis, the distance consultations can also be a convenient solution. The implementation of the telemedicine information systems is done via internet using open-source software; it is accessible both on $\mathrm{PC}$ and mobile devices and requires a broadband connection.
\end{abstract}

Keywords: Telemedicine, Amputated Limbs, Information Systems

\section{Introduction}

Telemedicine implies the use of information technology in order to exchange medical information from one site to another via electronic communications and its goal is to improve a patient's clinical health status. Telemedicine includes a growing variety of applications and services using two-way video, email, smart phones, wireless tools and other forms of telecommunications technology. Such applications of information technology in healthcare services can eliminate barriers regarding physical distances between patients and healthcare providers and can extend medical services in effective time to areas otherwise poorly or occasionally covered, like remote rural locations, isolated communities or countries with deficient medical infrastructure. As both telecommunications and medical investigation tools are domains of major scientific and technical research and development, telemedicine is rapidly progressing nowadays all over the globe and is expected to become in the next period a standard procedure both in developed and underdeveloped countries. The main services provided by telemedicine systems are emergencies support, medical consultations, remote patient monitoring, health information and medical education.

The health status of the population largely depends on the quality of medical services, on the effectiveness of interventions and on the extent to which a large number of patients can simultaneously be examined and treated. In EU countries, important efforts focus on increasing the care levels for disabled people, while maintaining the expenditures at tolerable levels for society. Public health is an issue that requires a constant attention because it directly influences all workforce categories, who, in their turn, generate progress in society. 
This paper describes a telemedicine system developed in order to support and improve effective healthcare for patients with amputated limbs and is a continuation of previous work (the design of myoelectric prosthesis with bio-feedback). The interest in this field is justified by the high number of cases of amputations. At international level, the number of people who had undergone forearm amputations is not precisely known, but statistics in recent years show that the number of patients with amputated arms worldwide is alarmingly high and it is constantly increasing: 450,000 in 2010 in the U.S. [1], 45,000 persons in the UK, 55,000 in Romania [2]. The 2010 statistics by disabled patients' organizations in the U.S. estimate that each year there are over 185,000 amputation procedures performed in the United States [3], [4]. The actual situation of such numerous people is difficult and constitutes a real concern. Such patients are confronted with great difficulties both in finding a job, for getting themselves reintegrated into society, and in performing daily personal hygiene activities, nutrition, etc., being often dependent on the financial support of their family and the government.

All over the world, in recent years, intelligent prostheses have enjoyed intense focus of attention, which is visible in the ever increasing number of international research projects, articles in scientific journals worldwide, and, last but not least, in commercial achievements such as the artificial hands with neuronal or myoelectric command [5], [6]. Systematic efforts made by many inter-disciplinary specialist research teams lead to significant annual progress that enriches the market with better performing artificial hands.

From practical perspectives, an artificial hand is expected to act similarly to the limb it replaces, inasmuch as possible, not only in movement possibilities and control, but also in point of sensory experience [9]. However, the best performing artificial hands manufactured nowadays are far from reaching the abilities of a biologically healthy hand. Still unsurpassed technological obstacles (bottlenecks) in achieving artificial hands fall into the next categories:

1. Limitation in the degrees of movement freedom and complexity. The best performing artificial hands on the current market have reached up to 15 degrees of freedom out of the 30 possessed by a healthy hand [12].

2. Limitation of the patient's voluntary control on the performed movement. In order to enable higher effectiveness in the patient's command of the prosthesis, certain researchers focus on conjugated collection and processing of numerous categories of bio-signals: neuron signals from the motor cortex, neuron signals from the peripheral nervous fibers, myoelectric intramuscular and surface signals [13]. Obtaining neuron commands (cortical or peripheral) requires surgical implantation of sensory matrices. This method has the advantage that it can offer multiple bio-signals with reduced noise, but it has the disadvantage of being invasive and its risks have not been assessed yet. Obtaining surface myoelectric signals (sEMG) is simple and non-invasive, but limited with respect to the number and quality of distinct signals to be gathered at stump level. Specialized scientific literature has reported several results in recognizing signal patterns and using them for achieving complex movements which would normally require a more substantial number of command signals [14].

3. Absence of complex sensorial feedback from the artificial hand to the patient. Implementing technical solutions in prostheses that will enable sensing temperature differences, touch and amounts of pressure felt during contact with various objects still remains one of the most important goals to be attained in the near future. At present, such functions are virtually absent in the products on the market [12]. Hence, one of the main limitations of artificial hands/arms is the lack of feedback that would be useful both for control and adjustment of the dynamic force exerted and also for the development of new reflexes in patients.

In recent years, the field of rehabilitation systems that use virtual reality has undergone continuous development in EU countries, the U.S. and Japan. Numerous interdisciplinary teams formed by experts perform research in the area of virtual reality technologies and thus generate progress in this area annually. The widely known institutes specialized in achieving rehabilitation systems based on virtual reality are located in Taiwan, Japan, the USA, Germany and the UK. Best achievements are currently in the research stage. Touch Bionics is the only company that now delivers a specialized software package "virtual-limb" with its "i-limb ultra" and "i-limb digits" prostheses. However, their product "virtual-limb" has reduced performance because it only offers palm and fingers modelling in virtual reality, completely lacking forearm, elbow and arm modelling.

The best prostheses and rehabilitation systems on the market are very expensive and most of amputees cannot afford such a product. New artificial hand models with good performances at lower costs will always be of great interest in this field. A multidisciplinary team of researchers, engineers and doctors form Romania proposed a new model of artificial hand [8], [18], [19]. Based on myoelectric signals and including different mechanisms of feed-back, this prosthesis need a systematic training effort for the patient, that implies in different stages both technical and medical assistance. The objective of the work reported in this paper is to provide an efficient training and communication tool for both patients and doctors to assist the patient rehabilitation effort. The rehabilitation training process is based on virtual reality and bio-feedback models. The telemedicine information system is a basic tool in the process of recovery and training of the patients, and can be extended for more general purposes.

\section{General Presentation of the Telemedicine System}

The telemedicine system is depicted by the overview image in figure 1. 


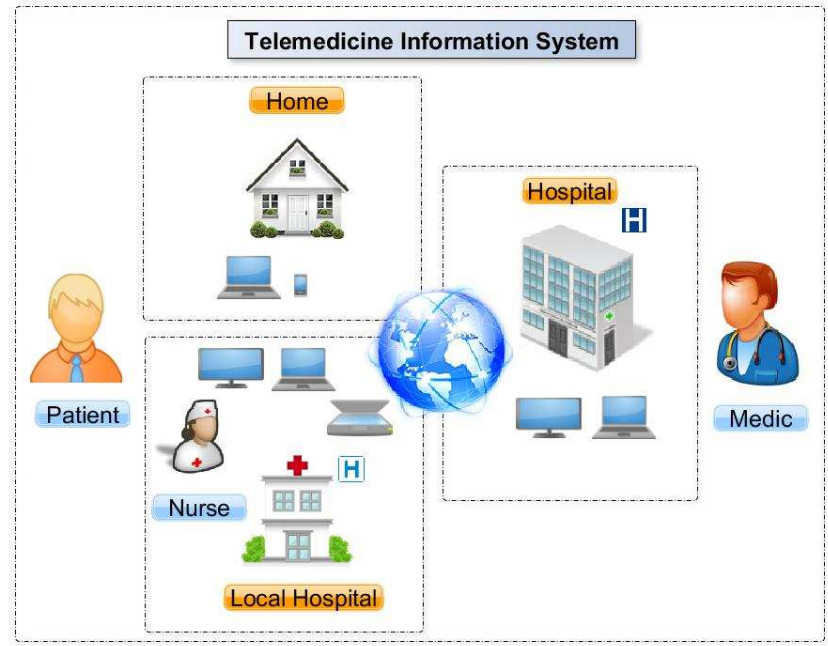

Figure 1. Overview of the telemedicine system.

The role of the telemedicine system implies the patient-medic direct communication either from home or from another hospital or healthcare center than the main hospital (where the doctor is located). The proposed information system uses the internet as telecommunication structure and requires access to a broadband internet connection for both the doctor and the patient. Basic knowledge regarding the usage of the home computer or mobile computing devices is necessary.

A telemedicine information system is easy to implement and presents benefits for the medical system, for the patient and for the doctor. The advantage for the medical system would be the ease of use for the electronic health file, the efficient management of hospital appointments. The doctor can schedule his patients according also to their possibilities and needs and will always have an extensive immediate overview over the patients' medical history.

The patient will have the possibility to "meet" the specialized doctor in his own house, which will increase the efficiency of consultations avoiding each time a stressful (especially for amputees) displacement. The patient has the possibility to schedule an online meeting with the doctor, according to a predefined timetable. The consultation will begin and end according to the schedule and momentary needs. The patient may attend an assisted training session at a medical center in his proximity and the session can be supervised by his doctor.

The necessity of an internet connection is not considered to be a limitation of the system. For instance, according to official a study of the National Statistics Institute from 2013, $55.8 \%$ of the households in Romania have a computer, from which $70.9 \%$ are in urban environment. Half of the households $(52.9 \%)$ have internet connection and the majority $(73.2 \%)$ are in urban areas [2]. Due to the fact that more than half of the population already possesses various knowledge regarding the usage of internet technology, the proposed system only adds a new utility to the suite of applications already being used on personal computers.

The proposed system offers an audio-video solution for consultation sessions. Therefore it requires a video camera, a microphone and speakers (headsets), usually embedded in laptop or mobile systems.

The interface providing interaction with the user is as simple as possible and makes use of well-known browser capabilities (Opera, Chrome, Firefox, etc.). Each user has an account, based on the email address and personal identification data.

Broadband connection is preferable for the proper use of the audio and video capabilities, this type of connection being more and more accessible lately in Romania as in most countries around the globe.

\section{Architecture and Design Considerations for the Telemedicine System Implementation}

The telemedicine system uses the classical approach of a client-server system comprising modern web-programming techniques for the server and for the client applications. The block diagram of the client-server system is presented in figure 2. The system is secured in order to provide confidentiality of the information and of the communication. The design of the architecture and its implementation is based on a modular concept, in order to give opportunities of further development.

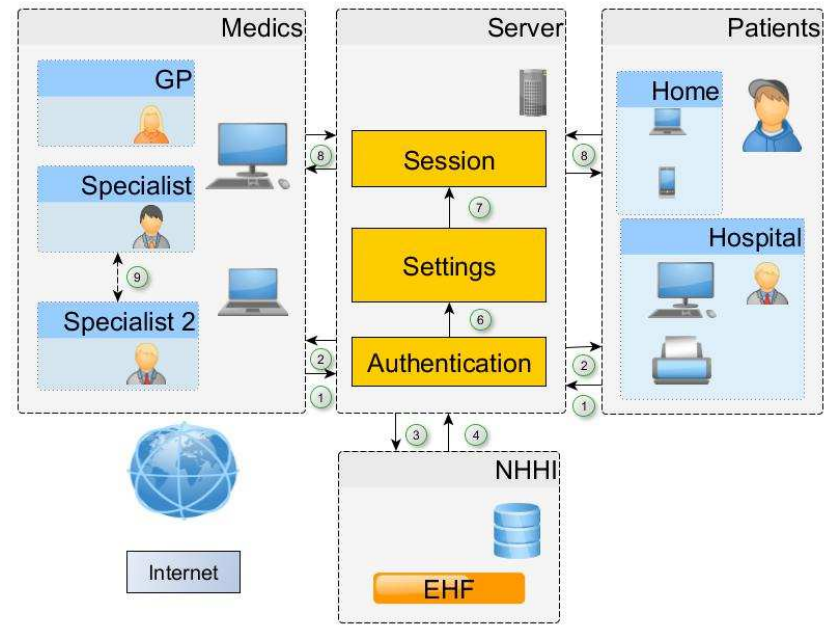

Figure 2. Block diagram of the telemedicine system.

From the perspective of the system designer, the doctor and the patient are both clients, although the interfaces and some functions may differ. All clients must be registered in the system in order to use it. The system will keep track of all connections for further reference.

The server application comprises three parts: the authentication module, the settings and data processing module and the session module.

The authentication module verifies, based on email and password, if the user is already registered in the system. If yes, based on the personal identification number, the electronic medical dossier is inquired. 
This module functions as a client for the National Assurance Agency and has the important task to interrogate the electronic file of the patient, but also to allow access to certain items of this file for the doctor. Also the adding of new data to the medical file is possible, if required. The settings and data processing module is the control module of the server. Local and personal data are sent to the doctor interface and also the scheduling and preparation of a consultation is taken care of. The local data refers to reservations and other system specific settings: registration dates, system access history or previous sessions. The session module links the doctor to the patient during a session. A data and an audio-video stream opens between the two users.

The patient can access the system from home as well as from a hospital or a recovery center, where a specialized assistant can also attend the consultation. The assistant can supervise and implement specific movements and exercises as well as measurements taken with specialized equipment. In order to be consulted, the patient must first schedule an online meeting according to the doctor's calendar. The doctor will have to confirm first the online consultation.

The doctor can access the consultation session practically from anywhere and can also set his availability to consult patients. He also can cancel requests if by some reason he cannot participate.

The system is meant to be used by a relative reduced number of clients, compared to search engines, social sites, online news, etc. and does not require extensive hardware and software resources.

The complexity of the system is given by the number of doctors and patients registered. Even if a large number of patients would like to schedule a consultation, the number of the doctors is limited and their timetables are fixed.

The most resource demanding component is the audio-video streaming. From the software point of view, this application was realized using only open-source software, covering all the functionality.

For the server part, the CentOS is an excellent solution as web server. The operating system hosts a LAMP (Linux Apache Mysql Php) platform that offers all necessary resources for a functional web hosting.

The client and interface modules use modern frameworks for a better user experience and also portability. Bootstrap is a front-end framework, modern and versatile, that offers the possibility to create with ease responsive web pages.

The audio-video module is covered by WebRTC protocol, that is supported by Google and will most likely soon become a wide spread protocol. It does not need a special plug-in (like Flash) to run.

\section{User Interface for the Patient and for the Doctor}

The user interface is as simple as possible, with a clear design and little distracting elements. The patient will perform three simple steps in order to attend a consultation: login into the system, scheduling and finally "attend" the consultation.

The registration into the system is done only once, by means of email address, password (used consequently for logging in) and personal ID number. The email address will also be used for further notifications. The next accessed page will be the homepage, which comprises only two menus: Reservation (consultation scheduling) and Consultation. Figure 3 reproduces the window for the Reservation (consultation scheduling) interface.

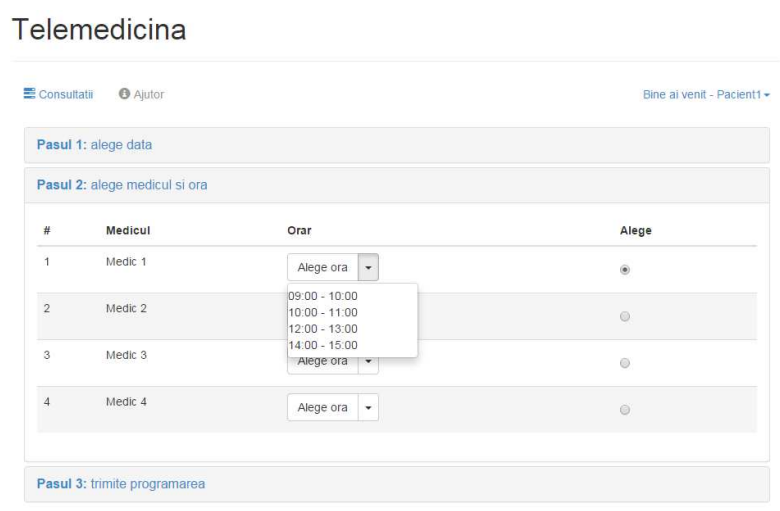

Figure 3. Reservation interface for the patient.

The doctor registration interface is slightly different, because the doctors need to be checked and validated. The doctor also logs in based on email and password and has access to his personalized page.

The consultation page has three menus: Consultation timetable creation, Consultation listing and Consultation.

The consultation is the final and most important step of the process. The interface is similar for doctor and patient. Figure 4 and 5 show the interface of a doctor that consults a patient from a rehabilitation center. This images are taken from an actual training session for the reactivation of the amputee forearm of a patient.

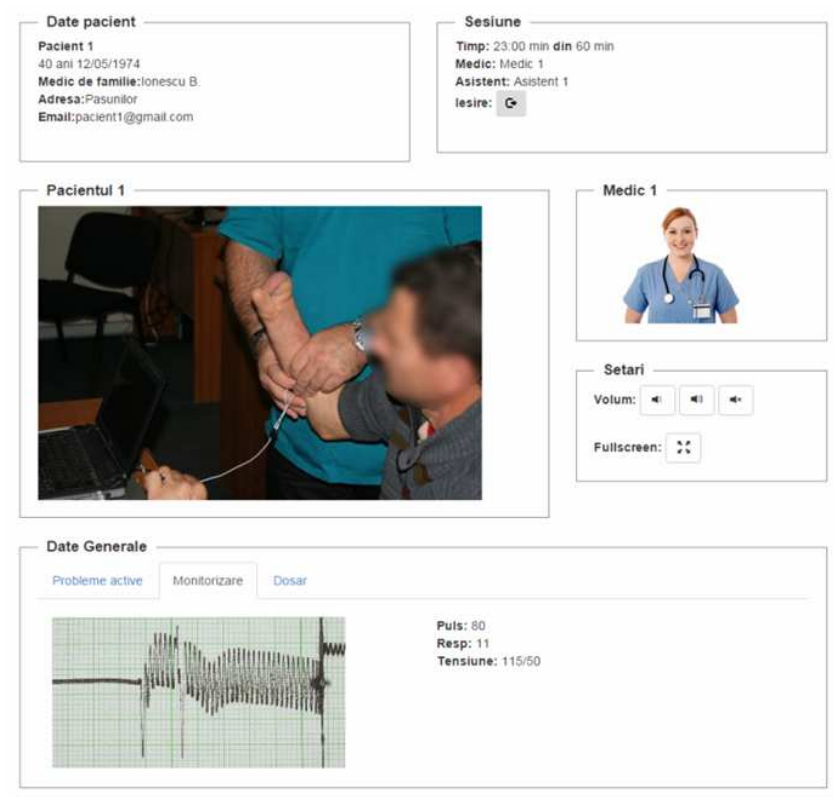

Figure 4. Consultation interface for the medic. 


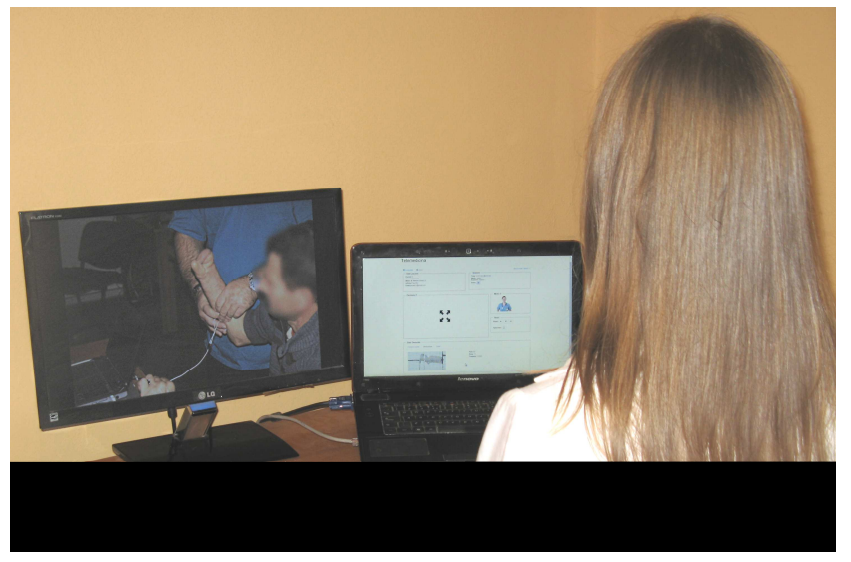

Figure 5. Consultation session.

If the patient would be at home, the live monitoring data would be missing. The patients interface shows the doctor in the live image.

The "Patient data" box presents general data regarding the patient.

"Session" displays the elapsed time, and the doctor's name.

"Patient data" offers detailed information about the patient, extracted from the electronic medical file and data acquired in real time by medical equipment.

\section{Functionality and Functional Scenarios}

Considering the interface describe above, we present here different scenarios, relating them to the general functionality of the system. Once validated, the doctor can see all consultations scheduled by patients. He can also deny any scheduling. If necessary, the doctor can set different reminders for the consultation.

When the patient $\log$ in, if he has made a previous reservation (consultation scheduling), a message will appear under the Consultation menu. The patient has the possibility to cancel the appointment, according to the policy of the hospital.

The consultation scheduling comprises three steps, the first being choosing the date of the consultation. Here a list of available doctors pops up and one of them can be chosen. Also a time interval will be reserved, as available for each specific doctor.

This is a dynamic list, depending on the doctors' timetable and on previous reservations. Figure 3 from the previous section depicts one step of the scheduling process.

The consultation will then occur at the scheduled time, when both the patient and the doctor will be online.

Figures 6 and 7 reproduce the functional diagrams of the telemedicine system. The functionality of the system implies two phases: scheduling and consultation, as seen in these figures. The main phase of the process and the most resource demanding is the audio-video consultation.

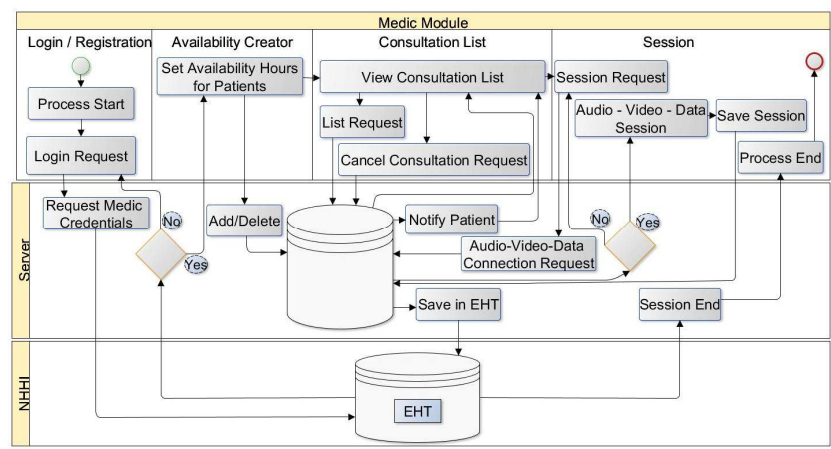

Figure 6. Functional diagram for doctor module

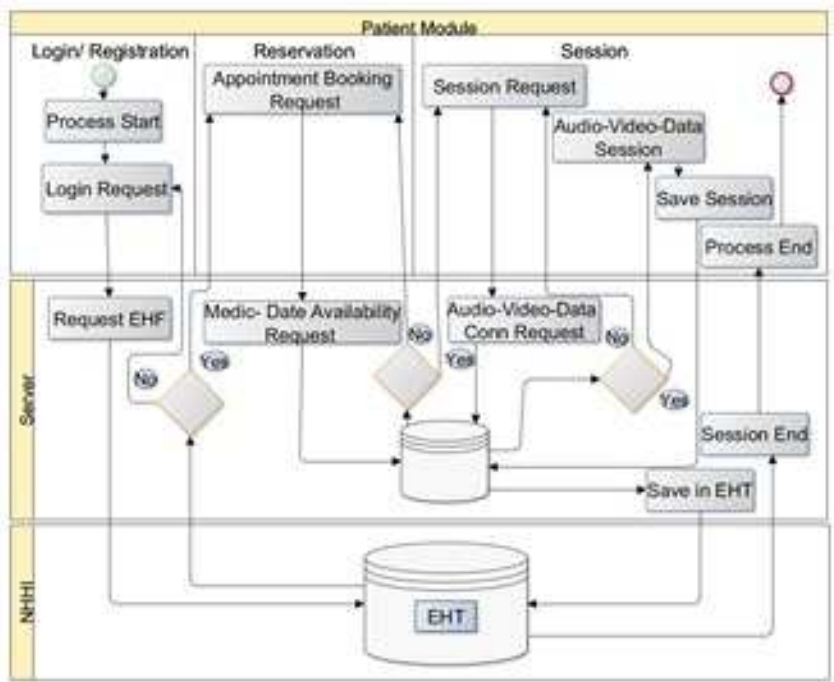

Figure 7. Functional diagram for patient module

The system presented here was developed for a special purpose but can be also used for general purposes. Currently the medical systems of numerous countries face critical shortage of public resources and significant lack of specialized personnel (nurses and specialist doctors). The health care system as well as the health care providers are compelled to provide the highest possible quality, for bringing profits to the clinic, under the difficult circumstances in which the National Health Insurance allocations are reduced by the government and the costs of health services per patient are rising. Under such circumstances, it becomes obvious that alternative modalities should be developed in patient care, by improving the standards for disabled people, while maintaining tolerable expenditure levels for the society. In order to solve these drastically conflicting demands, various equipment and techniques may be used, including informatics and telecommunications components that provide increased effectiveness in treating patients: the same supervising physician may care, in similarly proper conditions, for more patients, hence the cost is reduced (without lowering the care standards); the doctor is thus relieved from the duty of being permanently present at the patient's side. The telemedicine systems are therefore important from many perspectives. 


\section{Conclusions}

The telemedicine information system presented in this paper is a remote rehabilitation system with specific advantages. The patient can benefit from a consultation from home or another site where a doctor is not present. He will not have to displace each time he wants to contact a doctor, which is particularly beneficial for amputees.

The technical solution comprises open-source software and implements a simple interface that is easy to use and also effective for the aimed task. The steps needed to access an online consultation are few and similar to usual application procedures. The web solution is responsive and therefore portable, being accessible on home computers or tablets (or smartphones) with any operating system supporting a browser. The speed is limited only by the quality of the internet connection of the users.

The main specific advantage of the telemedicine system presented in this paper is the high accessibility at a training program offered to the amputee patients. These patients, in most cases, have a difficult financial situation and the performant (intelligent) prostheses are too expensive for them. Myoelectric prostheses can offer an affordable solution, but they imply a long training process. The patients that will use this system will join a program of rehabilitation that will help them learn to control the muscles and to use a myoelectric prosthesis.

\section{Acknowledgements}

"This work was supported from ARTHAND project (UEFISCDI), project number PN-II-PT-PCCA2013-4-0668."

\section{References}

[1] Amputee Coalition of America. 2010. ACA's Limb Loss Task Force warns of increasing limb loss in the U.S., O\&P Business News;6/1/2010, Vol. 19 Issue 7, p50, June 2010

[2] National Organization of Disabled People in Romania (ONPHR) - www.onphr.ro

[3] Martin Twiste, Limbless Statistics Annual Report: 2010/11,

[4] Jibby E. Kurichi, Barbara E. Bates, MD, Margaret G. Stineman, Amputation, International Encyclopedia of Rehabilitation, 2010

[5] David Pope, DARPA Prosthetics Programs Seek Natural Upper Limb, Neurotech reports, 2011

[6] Aleccia J. Limb loss a grim, growing global crisis, in NBC News, May 7, 2010

[7] Ziegler-Graham K, MacKenzie EJ, Ephraim PL, Travison TG, Brookmeyer R., Estimating the prevalence of limb loss in the United States: 2005 to 2050, Archives of Physical Medicine and Rehabilitation Volume 89, Issue 3 , Pages 422-429, March 2008
[8] Adrian Zafiu, et al, Modeling and Control System for Intelligent Prosthesis Configuration and Testing, Proceedings of The 9th International Conference on Computational Intelligence, Man-Machine Systems And Cybernetics (CIMMACS '10), pp. 265-268, Merida, Venezuela December $14-16,2010$

[9] Global Industry Analysts, Inc., Orthopedic Prosthetics - A Global Strategic Business Report (MCP-3224), March 2012

[10] Nicole Lewis, Global Telemedicine Market Headed For \$27 Billion, in BCC Research Market Forecasting, March 21, 2012

[11] Emily Delzell, Global lessons improve amputation prevention, Lower Extremity Review, June 2011, http://lowerextremityreview.com/article/global-lessons-improv e-amputation-prevention

[12] Aimee Schultz, Todd Kuiken, New Prospects for Prosthetics, Science in Society, March 2009

[13] Liat Clark, First mind-controlled, bone-mounted robotic arms to be implanted in 2013, Wired Science, November 12

[14] RSL Steeper, The bebionic 3 myoelectric hand product features - bebionic http://bebionic.com/the_hand/features

[15] Burck JM, Bigelow JD, Harshbarger SD, Revolutionizing Prosthetics: Systems Engineering Challenges and Opportunities, Johns Hopkins APL Tech Dig, 30(3):186-197 (2011)

[16] FP7 EU Research Projects, Wearable interfaces for hAnd function recoverY (WAY), 2011-2014, http://cordis.europa.eu/projects/rcn/100704_en.html

[17] Loredana Stanciu, Antonius Stanciu, Designing and Implementing a Human Hand Prosthesis, IFMBE Proceedings, International Conference on Advancements of Medicine and Health Care through Technology, Cluj-Napoca, Sept., 2009, p. 399-404, ISBN: 978-3-642-04291-1, ISI Proceedings

[18] Lucian Milea, Gheorghe Stefan, Adrian Barbilian et al., Control System for Locomotory Prosthesis Configuration, International Journal Of Systems Applications, Engineering \& Development, Issue 3, Volume 5, 2011, pp. 420-427

[19] Lucian Milea, Gheorghe Stefan, Adrian Barbilian, Ion Codorean, Marius Moga, Stefan Mitulescu, Valeriu Codreanu, Tiberiu Boros, Mihai Teodorescu, Control System for Locomotory Prosthesis Configuration, International Journal Of Systems Engineering, Applications And Development, Issue 1, Volume 5, March 2011, pp. 420-427, ISSN: 2074-1308, http://www.universitypress.org.uk/journals/saed

[20] Grant McGimpsey and Terry C. Bradford, Limb Prosthetics Services and Devices Critical Unmet Need: Market Analysis, Bioengineering Institute Center for Neuroprosthetics Worcester Polytechnic Institution,

[21] Ziegler-Graham K, MacKenzie EJ, Ephraim PL, Travison TG, Brookmeyer R., Estimating the prevalence of limb loss in the United States: 2005 to 2050, Archives of Physical Medicine and Rehabilitation Volume 89, Issue 3 , Pages 422-429, March 2008

[22] Chiharu Ishii, Recognition of Finger Motions for Myoelectric Prosthetic Hand via Surface EMG, in Advances in Mechatronics, ISBN 978-953-307-373-6, Published: August 29, 2011, 176 - 190, DOI: 10.5772/21812 
[23] Claudio Castellini, Angelo E Fiorilla, and Giulio Sandini Multi-subject/daily-life activity EMG-based control of mechanical hands, Journal of NeuroEngineering and Rehabilitation 2009, 6:41 doi:10.1186/1743-0003-6-41

[24] Miller LA, Stubblefield KA, Lipschutz RD, Lock BA, Kuiken TA: Improved myoelectric prosthesis control using targeted reinnervation surgery: A case series, IEEE Trans Neural Syst Rehabil Eng 2008, 16:46-50

[25] Sachpazidis, Ilias (10 July 2008). Image and Medical Data Communication Protocols for Telemedicine and Teleradiology (dissertation). Darmstadt, Germany: Department of Computer Science, Technical University of Darmstadt.
[26] George R. Schwartz, C. Gene Cayten; George R. Schwartz (editor). Principles and Practice of Emergency Medicine, Volume 2, Lea \& Febiger, 1992, pg.3202, ISBN 0-8121-1373-X, ISBN 978-0-8121-1373-0.

[27] Rogove, Herbert J.; McArthur, David; Demaerschalk, Bart M.; Vespa, Paul M. (January-February 2012). Barriers to Telemedicine: Survey of Current Users in Acute Care Units. "Telemedicine and e-Health". Telemedicine and e-Health 18 (1): 48-53. doi:10.1089/tmj.2011.0071. PMID 22082107. 